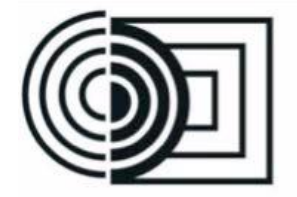

$16^{\circ}$ Ergodesign - Congresso Internacional de Ergonomia e Usabilidade de Interfaces Humano Tecnológica: Produto, Informações Ambientes Construídos e Transporte

$16^{\circ}$ USIHC - Congresso Internacional de Ergonomia e Usabilidade de Interfaces Humano Computador

CINAHPA | 2017 - Congresso Internacional de Ambientes Hipermídia para Aprendizagem.

\title{
REVISÃO INTEGRATIVA SOBRE INTERFACES DIGITAIS PARA SELEÇÃO TIPOGRÁFICA
}

\author{
INTEGRATIVE REVIEW ABOUT DIGITAL INTERFACES \\ FOR TYPOGRAPHICAL SELECTION
}

\author{
Mary Vonni Meürer ${ }^{1}, \mathrm{Me}$ \\ Berenice Santos Gonçalves², Dra \\ (1) Universidade Federal de Santa Catarina \\ e-mail: mary.meurer@ufsc.br \\ (2) Universidade Federal de Santa Catarina \\ e-mail: berenice@cce.ufsc.br
}

Tipografia, Seleção Tipográfica, Interfaces Digitais

\begin{abstract}
Este artigo apresenta o resultado de uma pesquisa de Revisão Integrativa realizada com o objetivo de identificar autores e pesquisas relacionadas ao desenvolvimento ou análise de interfaces digitais para seleção tipográfica. A pesquisa foi realizada nas principais bases de dados da área de Design usando as palavras chave "typeface" e "selection" e retornou 25 artigos. Após análise dos resumos destes artigos, apenas 3 foram considerados relevantes para a pesquisa, sendo que apenas 1 interface digital citada nos artigos está concluída e em funcionamento. Desta forma, observa-se que o assunto tem sido pouco citado e que existe espaço para mais pesquisas e desenvolvimentos nesta área. Integra-se a esta pesquisa a revisão bibliográfica que resultou em 8 critérios preliminares de seleção tipográfica: legibilidade, variações e recursos, aspectos histórico-culturais, expressão, qualidade, suporte, licenciamento e investimento. Ao comparar os recursos oferecidos pelas interfaces citadas nos artigos com os critérios definidos, constatou-se que estas trouxeram contribuições relevantes. A seleção por atributos formais e de personalidade da fonte, por exemplo, está contemplada. Porém, não é possível selecionar por meio destas interfaces fontes de acordo com suas regras de licenciamento, extensão da família e presença de caracteres específicos, como sugerem os critérios resultantes da revisão bibliográfica.
\end{abstract}

\section{Typography, Typographical Selection, Digital Interfaces}

This article presents the results of an integrative review research carried out with the objective of identifying authors and research related to the development or analysis of digital interfaces for typographic selection. The research was carried out in the main databases of the Design area using the keywords "typeface" and "selection" and returned 25 articles. After analyzing the abstracts of these articles, only 3 were considered relevant for the research, and only 1 digital interface cited in the articles is completed and in operation. In this way, it is observed that the subject has been little mentioned and that there is room for more research and developments in this area. It integrates to this research the bibliographic revision that resulted in 8 preliminary criteria of typographic selection: legibility, variations and resources, historical-cultural aspects, expression, quality, support, licensing and investment. When comparing the resources offered by the interfaces mentioned in the articles with the defined criteria, they were found to have brought relevant contributions. Selection by formal attributes and personality of the source, for example, is 


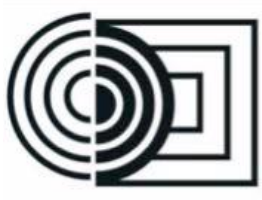

$16^{\circ}$ Ergodesign - Congresso Internacional de Ergonomia e Usabilidade de Interfaces Humano Tecnológica: Produto, Informações Ambientes Construídos e Transporte

$16^{\circ}$ USIHC - Congresso Internacional de Ergonomia e Usabilidade de Interfaces Humano Computador

CINAHPA | 2017 - Congresso Internacional de Ambientes Hipermídia para Aprendizagem.

contemplated. However, it is not possible to select through these interfaces sources according to their licensing rules, family extension and presence of specific characters, as suggested by the criteria resulting from the bibliographic review.

\section{Introdução}

A tipografia está presente em praticamente todas as atividades do designer gráfico como: desenvolvimento de projetos editoriais, de interface, embalagens, identidade visual, sinalização, entre outros. Além disso, a própria criação de fontes e famílias tipográficas também é uma atividade desenvolvida pelo designer. Segundo Farias (2013) o termo tipografia envolve o design de tipos, a sua aplicação no projeto gráfico ou o design com tipos e também o processo de reprodução tipográfica.

No que se refere ao design com tipos, uma das dificuldades encontradas logo no início do processo é a escolha das fontes, ou conjunto de fontes tipográficas que serão usadas no projeto. Como observa Bringhurst (2015), embora atualmente um tipógrafo possa ter acesso a milhares de fontes digitais por meio da internet, as suas seleções parecem sempre incompletas. Hendel (2003) concorda com Bringhurst ao afirmar que o número de fontes disponíveis atualmente dificulta a escolha.

Hendel (2003) observa que a expansão da produção tipográfica ocorreu porque a era digital permitiu que as fontes fossem produzidas com mais rapidez por eliminar as etapas de produção como matrizes, moldes e fundição. As fontes digitais consequentemente tem um custo significativamente menor do que os tipos de metal, podendo ser adquiridas com mais variedade. Unger (2016) comenta que os avanços tecnológicos ocorridos a partir da década de 1980 contribuíram para acelerar o processo de introdução de novas fontes no mercado em quantidades inéditas.

Diante de tantas opções disponíveis, como escolher bem as fontes tipográficas para cada projeto?
Segundo o guia Typography Primer (2000) ${ }^{1}$ publicado pela Adobe $\odot$, diversos fatores como público-alvo, valores estéticos, tom da mensagem e meio de publicação, influenciam na escolha do tipo. Para Ali (2009) as escolhas em tipografia são incertas e existem muitas incógnitas e variáveis que precisam ser trabalhadas. A autora afirma que é preciso um longo período de estudo e aplicação para se ter domínio sobre o assunto. É necessário estabelecer critérios para reconhecer qual a fonte ideal diante de tantas possibilidades.

Partindo das contribuições oriundas de autores como Leeuwen (2006), Frutiger (2007), Jury (2007), Ali (2009), Beier (2009), Samara (2010), Frascara (2011), Fontoura e Fukushima (2012), Rocha (2012), Farias (2013), Pamental (2014), Zapaterra (2014), Bringhurst (2015), Lupton (2015), Smeijers (2015) e Unger (2016) foram definidos 8 critérios de seleção tipográfica. Estes critérios foram categorizados segundo fatores apontados por Henestrosa, Meseguer e Scaglione (2014) resultando na versão preliminar de um conjunto de critérios para seleção tipográfica que compreende: fatores formais e funcionais, fatores estéticos, fatores técnicos e fatores legais e econômicos, como mostra o quadro 1 .

\begin{tabular}{|l|l|}
\hline \multicolumn{1}{|c|}{ Categoria } & \multicolumn{1}{c|}{ Critérios } \\
\hline $\begin{array}{l}\text { Fatores } \\
\text { formais e } \\
\text { funcionais }\end{array}$ & $\begin{array}{l}\text { Legibilidade: formas dos caracteres e sua } \\
\text { adequação aos padrões de leitura de acordo } \\
\text { com o público-alvo }\end{array}$ \\
\hline
\end{tabular}

${ }^{1}$ O Typography Primer, ou Adobe TypePrimer, é um guia de referência disponibilizado pela empresa em seu site no link http://wwwimages.adobe.com/content/dam/Adobe/en/pr oducts/type/pdfs/adobe-type-primer.pdf Acesso em 30 de abril de 2016. 


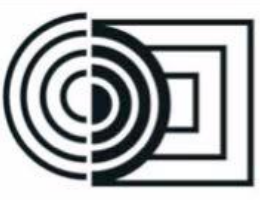

ERGODESIGN USIHC CINAHPA

\begin{tabular}{|c|c|}
\hline Categoria & Critérios \\
\hline & $\begin{array}{l}\text { Variações e Recursos: pesos e estilos que a } \\
\text { família tipográfica, a qual fonte pertence, } \\
\text { oferecem. }\end{array}$ \\
\hline \multirow[t]{2}{*}{$\begin{array}{l}\text { Fatores } \\
\text { estéticos }\end{array}$} & $\begin{array}{l}\text { Aspectos Histórico-Culturais: devem ser } \\
\text { considerados principalmente quando } \\
\text { espera-se que uma fonte represente } \\
\text { determinada época ou região }\end{array}$ \\
\hline & $\begin{array}{l}\text { Expressão: refere-se a percepção de } \\
\text { atributos de personalidade de acordo com o } \\
\text { desenho dos caracteres, considerando o } \\
\text { potencial expressivo dos tipos. }\end{array}$ \\
\hline \multirow[t]{2}{*}{$\begin{array}{l}\text { Fatores } \\
\text { técnicos }\end{array}$} & $\begin{array}{l}\text { Qualidade: os parâmetros e o desenho da } \\
\text { fonte devem ser adequados para garantir } \\
\text { uma boa renderização e/ou } \\
\text { impressão. }\end{array}$ \\
\hline & $\begin{array}{l}\text { Suporte: a fonte deve ser adequada ao } \\
\text { suporte onde será aplicada pois este } \\
\text { interfere diretamente na sua percepção. }\end{array}$ \\
\hline \multirow{2}{*}{$\begin{array}{l}\text { Fatores } \\
\text { legais e } \\
\text { econômicos }\end{array}$} & $\begin{array}{l}\text { Licenciamento: regras de uso e } \\
\text { customização da fonte definidas na licença. }\end{array}$ \\
\hline & $\begin{array}{l}\text { Investimento: adequação entre custo das } \\
\text { licenças e orçamento do projeto. }\end{array}$ \\
\hline
\end{tabular}

Quadro 1: Conjunto preliminar de critérios para seleção tipográfica

Fonte: Elaborado pelas autoras

Os critérios apresentados no quadro 1 constituem uma pesquisa mais ampla, e ainda em desenvolvimento, sobre o processo de seleção tipográfica.

A pesquisa apresentada neste artigo teve como objetivo buscar referências sobre o desenvolvimento de ferramentas ou interfaces digitais que tenham relação com a seleção tipográfica. Os resultados da revisão sistemática foram confrontados com os critérios definidos, verificando assim se tais ferramentas ou interfaces poderiam ser usadas para selecionar fontes a partir destes critérios. $16^{\circ}$ Ergodesign - Congresso Internacional de Ergonomia e Usabilidade de Interfaces Humano Tecnológica: Produto, Informações Ambientes Construídos e Transporte

$16^{\circ}$ USIHC - Congresso Internacional de Ergonomia e Usabilidade de Interfaces Humano Computador

CINAHPA | 2017 - Congresso Internacional de Ambientes Hipermídia para Aprendizagem.

\subsection{Método de pesquisa}

A revisão integrativa possibilita a síntese de vários estudos já publicados, permitindo a geração de novos conhecimentos, pautados nos resultados apresentados pelas pesquisas anteriores (MENDES; SILVEIRA; GALVÃO; BENEFIELD; POLIT; BECK apud BOTELHO et al, 2011).

De acordo com Whitemore e Knafl (2005) a revisão integrativa pode contribuir para o desenvolvimento de teorias, pois, apresenta o estado da arte sobre determinado tema, incluindo estudos com diversas metodologias, experimentais ou não-experimentais. A realização deste tipo de revisão requer uma sequencia com determinadas etapas para garantir a sistematização do processo, permitindo, inclusive, que este seja repetido futuramente em busca de novos resultados.

Este estudo seguiu as 6 etapas propostas por

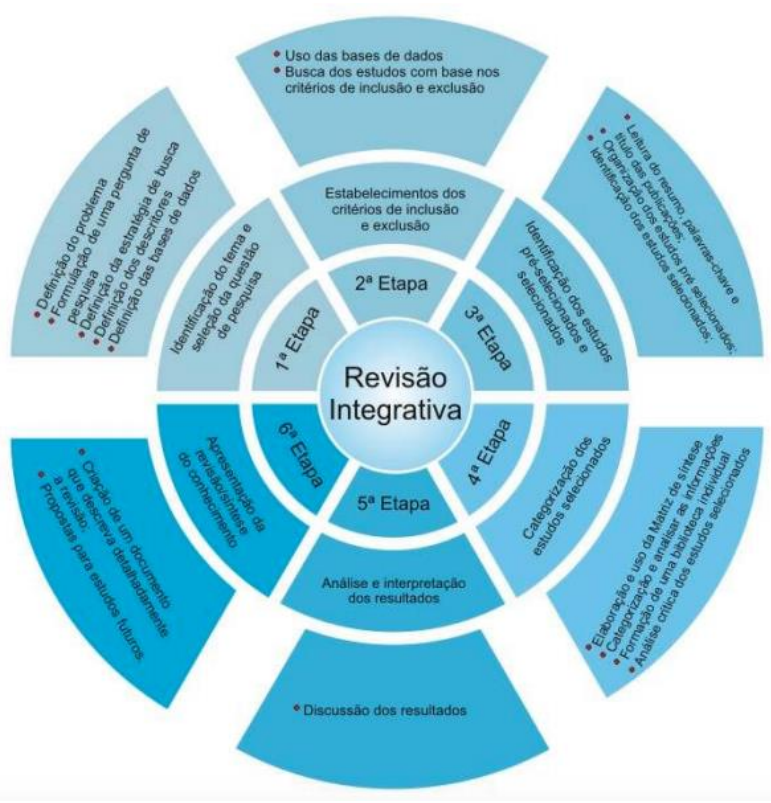

Botelho et al (2011), como mostra a figura 1.

Figura 1: Processo de Revisão Integrativa Fonte: Botelho et al (2011) 


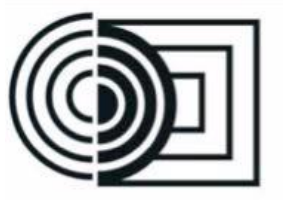

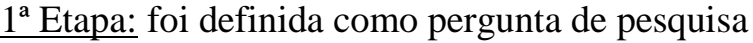
se existem ferramentas ou interfaces digitais que possam auxiliar no processo de seleção tipográfica. A estratégia de busca iniciou-se a partir das combinações dos seguintes termos: tools AND typeface AND selection, "digital interface" AND typeface AND selection, typeface AND (selection OR choose OR combination), typography AND tool* e typeface AND selection. A combinação de palavras-chave que apresentou melhores resultados foi typeface AND selection, resultando em um número mais significativo de artigos. As bases selecionadas foram Scopus, Science Direct, Scielo e Web of Science, consideradas as mais relevantes para a área de Design e a pesquisa foi realizada em dezembro de 2016.

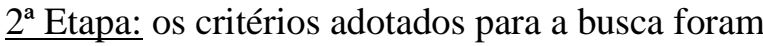
artigos nos idiomas inglês e espanhol, publicados a partir de 2005, priorizando, assim, por estudos mais recentes. Também foram priorizados artigos científicos e livros, descartando fontes como congressos, revistas não científicas, entre outros.

3a Etapa: Para a organização dos estudos préselecionados foi usado o gerenciador de arquivos Mendeley, a fim de otimizar o processo de seleção.

$4^{\mathrm{a}}$ Etapa: Após a leitura dos resumos muitos artigos foram descartados pois não apresentavam conteúdo relacionado que fosse significativo.

Como foram poucos os artigos restantes, apenas 3, não houve necessidade de uma matriz de síntese.

$5^{a}$ Etapa: Os artigos mais relevantes receberam especial atenção no processo de análise $\mathrm{e}$ interpretação. Nesta etapa houve a integração com as referências bibliográficas referentes a critérios de seleção de fontes.

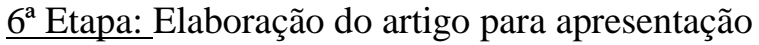
dos resultados obtidos com a pesquisa.

\section{Descrição do Processo}

Após definir a pergunta de pesquisa foram testadas diversas palavras-chave nas 4 bases de dados, conforme mencionado anteriormente. Buscando maior relevância nas respostas foi definido que as palavras-chave typeface AND selection deveriam $16^{\circ}$ Ergodesign - Congresso Internacional de Ergonomia e Usabilidade de Interfaces Humano Tecnológica: Produto, Informações Ambientes Construídos e Transporte

$16^{\circ}$ USIHC - Congresso Internacional de Ergonomia e Usabilidade de Interfaces Humano Computador

CINAHPA | 2017 - Congresso Internacional de Ambientes Hipermídia para Aprendizagem.

aparecer no título, resumo ou palavras-chave do artigo. O quadro 2 sintetiza o resultado das buscas.

\begin{tabular}{|l|c|c|}
\hline \multicolumn{1}{|c|}{ Base } & Total de Artigos & Relevantes \\
\hline Scielo & 0 & 0 \\
\hline Web of Science & 15 & 1 \\
\hline Science Direct & 1 & 0 \\
\hline Scopus & 9 & 2 \\
\hline
\end{tabular}

Quadro 2: Relação das bases consultadas e artigos encontrados

Fonte: Elaborado pela autora

Embora a pesquisa inicialmente fosse direcionada para ferramentas, este termo não foi usado pois não retornou artigos relevantes nos testes de palavraschave. Lendo os 3 artigos restantes observou-se que termos como interface e biblioteca de fontes são empregados para designar o que para esta pesquisa entende-se como ferramenta. Sendo assim assumiu-se dai em diante o termo interface digital como padrão.

A seguir são apresentados os aspectos mais relevantes dos 3 artigos e posteriormente os recursos das interfaces digitais analisadas são confrontados com os critérios definidos a partir da revisão bibliográfica.

\section{Análise de Resultados}

O primeiro artigo considerado relevante, Exploratory font selection using crowdsourced attributes (O'DONOVAN et al, 2014) enfatiza a importância da tipografia para o design e argumenta que a escolha de uma fonte é algo sutil e que os profissionais de design fazem cursos inteiros sobre tipografia para se preparar, mas para os novatos pode ser algo frustrante e obscuro. Buscando mais estudos relacionados aos autores constatou-se que a pesquisa apresentada no artigo resultou em uma tese intitulada "Learning Design: Aesthetic Models for Color, Layout, and Typography" defendida por O'Donovan em 2015. Analisando a tese correspondente concluiu-se que 


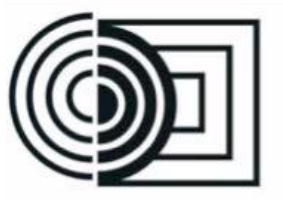

a parte relacionada a tipografia trazia o mesmo conteúdo já visto no artigo.

O'Donovan et al (2014) afirmam no artigo e na tese que a interface para a escolha de fonte nos softwares é a mesma há anos e que as enormes listas geradas induzem o usuário a escolher sempre as mesmas fontes, por padrão ou familiaridade. De acordo com autores, os desafios para selecionar fontes consistem em:

- Escolher entre tantas opções disponíveis.

- Não existir um método óbvio para categorizar os objetivos do usuário. Listagens organizadas por categorias como serifa ou display não correspondem necessariamente a estes objetivos e os nomes das fontes raramente são significativos.

- As tarefas que o usuário deseja realizar ao buscar a fonte podem ser diferentes, por exemplo procurar uma fonte com o estilo de uma imagem particular, identificar fontes gratuitas semelhantes a uma fonte paga, ou ainda explorar um grande conjunto de fontes como o do Adobe ${ }^{\circledR}$ TypeKit ou Google ${ }^{\circledR}$ Web Fonts (O’DONOVAN et al, 2014).

O'Donovan et al (2014) propuseram em seu estudo uma interface digital que permite a seleção de fontes de acordo com seus atributos, ou seja adjetivos que descrevem a fonte de acordo com sua aparência ou personalidade visual, como amigável, formal ou legível. O usuário pode selecionar vários atributos e o sistema cruza os dados mostrando fontes que atendem a estes atributos. Para a interface foram usados os 31 atributos de personalidade (dramático, delicado, elegante) definidos por Shaikh (2007) acrescidos de mais 6 atributos comumente usados por designers para descrever as fontes e que são relacionados a aspectos tipográficos como cursivo, display, itálico, etc.

Na segunda etapa propõe-se ao usuário fontes agrupadas de acordo com a sua aparência visual, de forma hierárquica. Na terceira etapa, após o usuário selecionar uma fonte, a interface apresentava a ele diversas fontes similares visualmente, para que ele pudesse escolher. As $16^{\circ}$ Ergodesign - Congresso Internacional de Ergonomia e Usabilidade de Interfaces Humano Tecnológica: Produto, Informações Ambientes Construídos e Transporte

$16^{\circ}$ USIHC - Congresso Internacional de Ergonomia e Usabilidade de Interfaces Humano Computador

CINAHPA | 2017 - Congresso Internacional de Ambientes Hipermídia para Aprendizagem.

figuras 2 e 3 ilustram a sequencia de telas apresentada.

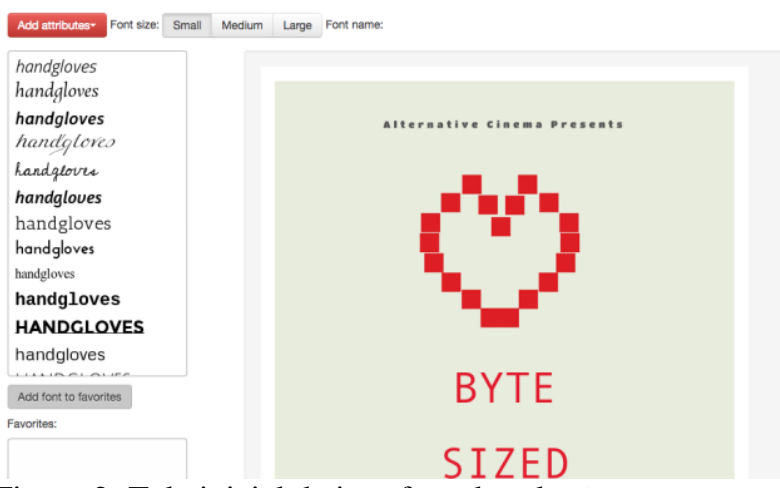

Figura 2: Tela inicial da interface de seleção. Disponível

http://www.dgp.toronto.edu/ donovan/font/

em:

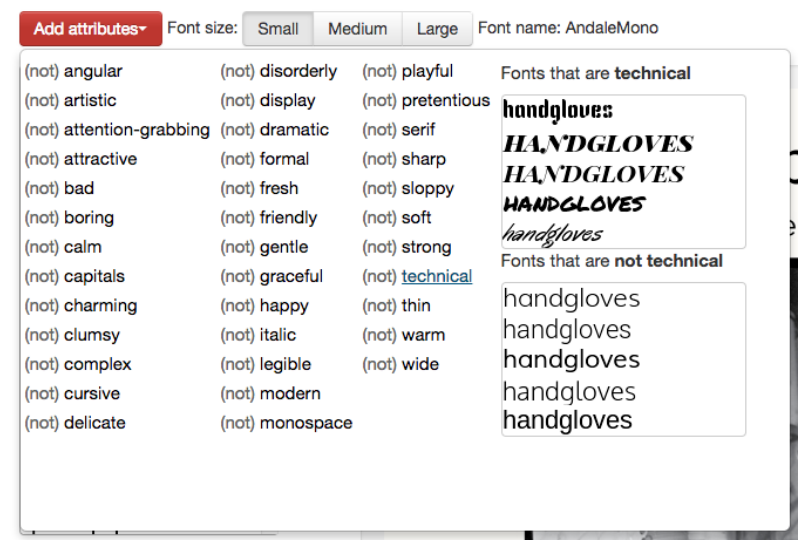

Figura 3: Detalhe do menu de seleção baseado em atributos da fonte.

Disponível em:

http://www.dgp.toronto.edu/ donovan/font/

Depois foram calculados dados a partir de um modelo baseado em crowdsourcing, ou seja, usando conhecimentos coletivos compartilhados pela internet. Com base nestes cálculos foi possível prever valores de atributos e semelhanças formais para que o sistema possa agregar novas fontes sem a necessidade de novos dados.

Com o protótipo finalizado foi realizada uma pesquisa com estudantes universitários para que eles usassem a interface com o objetivo de selecionar fontes para 15 layouts diferentes. A pesquisa utilizou a plataforma Amazon Mechanical Turk, que consiste em um espaço para realizar pesquisas que envolvam inteligência humana 


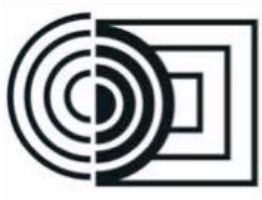

aplicada a tarefas que os computadores atualmente não são capazes de realizar. Os pesquisadores constataram que os usuários tinham mais facilidade para selecionar fontes usando a interface proposta do que as tradicionais listas de fontes. Mas destacaram que essa facilidade é mais significativa estatisticamente.

Em relação a outras interfaces comerciais para fontes, o artigo apresenta opções disponíveis no mercado que vão além das listagens, possibilitando um sistema de busca mais sofisticado, como o IdentiFont, o MyFonts, What the Font e Fontspring 2 . Destaca ainda o TypeDNA que permite a busca com base em 4 atributos (peso, largura, itálico ou óptico). Porém como tratam-se de códigos fechados relataram ser difícil fazer uma aproximação com a sua pesquisa.

Como desdobramentos futuros os autores argumentam que a interface de seleção de fontes também poderá ser usada em outras áreas onde os usuários precisem procurar em grandes conjuntos de dados, como a seleção de ilustrações vetoriais, por exemplo.

O'Donovan et al (2014) consideram que embora os atributos e semelhanças da fonte sejam propriedades inerentemente subjetivas existe um consenso entre as opiniões humanas para construir um modelo razoavelmente eficaz. Porém, apontam que uma limitação desta abordagem é o fato de se considerar sempre a média, descartando as escolhas divergentes. Técnicas de filtragem colaborativa são apontadas como uma possibilidade para a modelagem de indivíduos.

O sistema de busca IdentFont indicado no artigo anterior é o objeto de análise do segundo artigo, intitulado "Uma biblioteca de fontes online: avaliação por estudantes de design" da autoria de Chen e Choi (2006).

\footnotetext{
${ }^{2}$ Os sites citados no artigo estão disponíveis nos seguintes endereços: IdentiFont http://www.identifont.com/, MyFonts http://www.myfonts.com/, What the Font http://www.myfonts.com/WhatTheFont/, FontSpring https://www.fontspring.com/ e TypeDNA https://www.fontspring.com/. Acessados em 19 de março de 2017.
}

$16^{\circ}$ Ergodesign - Congresso Internacional de Ergonomia e Usabilidade de Interfaces Humano Tecnológica: Produto, Informações Ambientes Construídos e Transporte

$16^{\circ}$ USIHC - Congresso Internacional de Ergonomia e Usabilidade de Interfaces Humano Computador

CINAHPA | 2017 - Congresso Internacional de Ambientes Hipermídia para Aprendizagem.

Para desenvolver a pesquisa sobre a biblioteca de fontes - IdentFont - os autores selecionaram 35 alunos de graduação e pós-graduação de vários programas acadêmicos relacionados ao design da Universidade do Texas entre setembro de 2004 e abril de 2005. O objetivo era descobrir a lógica das questões propostas pela IdentFont no processo de pesquisa e seleção de fontes e a eficácia percebida pelos usuários.

De acordo com os autores, a pesquisa se faz necessária devido ao aumento de fontes disponíveis e as dificuldades em realizar uma busca diante de tantas opções.

\footnotetext{
"Com este aumento do volume de fontes disponíveis, no entanto, a tarefa de encontrar uma fonte específica torna-se mais difícil. As fontes são frequentemente classificadas em catálogos, mas este esquema requer que os usuários estejam familiarizados com a terminologia da fonte e as próprias categorias permanecem grandes. Cada fonte tem um nome exclusivo, mas poucas pessoas sabem o nome das fontes que procuram. Encontrar uma fonte específica, navegando por um grande catálogo, mesmo quando categorizado, pode ser muito demorado e cansativo." (CHEN e CHOI, 2006, tradução nossa)
}

No artigo os autores citam outras interfaces de gerenciamento de fontes como SearchFreeFonts, MyFonts e FontExpert, mas optam pela IdentFont. Esta interface corresponde a uma biblioteca de fontes online criada em novembro de 2.000 por uma consultoria de design da informação baseada em Cambridge, como foco da pesquisa.

A IdentFont é formada por um sistema especialista que possibilita a busca em um catálogo com milhares de fontes comercializadas por 200 fundições tipográficas. A busca pode ser feita pela aparência da fonte, pelo nome, pela similaridade com outras fontes, pela presença de determinados pictogramas ou ainda pelo nome dos designers de tipos, como mostra as figuras 4 e 5 . 


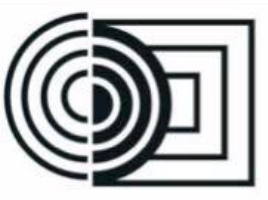

$16^{\circ}$ Ergodesign - Congresso Internacional de Ergonomia e Usabilidade de Interfaces Humano Tecnológica: Produto, Informações Ambientes Construídos e Transporte

$16^{\circ}$ USIHC - Congresso Internacional de Ergonomia e Usabilidade de Interfaces Humano Computador

CINAHPA | 2017 - Congresso Internacional de Ambientes Hipermídia para Aprendizagem.

satisfação com os registros de tela feitos no laboratório de usabilidade, onde foi possível observar o número de perguntas (passos) que os participantes percorreram para concluir cada tarefa. $\mathrm{O}$ artigo apresenta detalhadamente a análise dos dados, como mostra o gráfico da figura 6 .

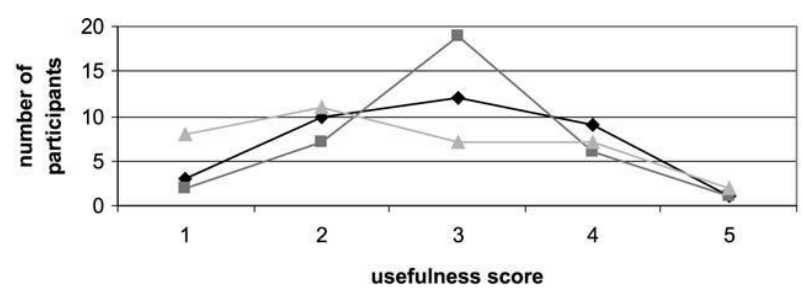

Do the characters have serifs?

Serifs are spikes or slabs on the ends of the strokes.
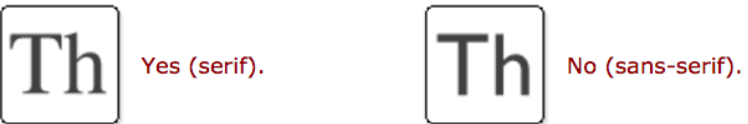

\section{Not Sure}

Figura 5: Detalhe da seleção pela aparência Disponível em: http://www.identifont.com/

O método de pesquisa do artigo consistiu em apresentar aos 35 usuários 6 tarefas que envolviam encontrar fontes a partir de um conjunto completo de caracteres, de imagens e também de conjuntos incompletos de caracteres. Neste último caso os participantes precisariam adivinhar as características dos caracteres faltantes tomando por base os outros caracteres.

Após completar as 6 tarefas os usuários deveriam responder a um questionário sobre sua satisfação com a ferramenta utilizando a escala Likert (de 1 a 5) e envolvendo questões sobre:

- a experiência dos participantes com design gráfico e computadores;

- uso do computador dos participantes;

- avaliação subjetiva da conclusão da tarefa;

- significado de procedimentos de tarefas; e

- utilidade da ferramenta online. (CHEN e CHOI, 2006, tradução nossa)

Os pesquisadores mediram o sucesso ou fracasso na realização da tarefa confrontando as informações obtidas nos questionários de

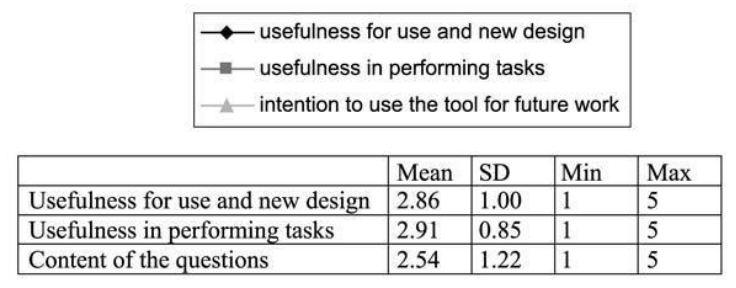

Notes: Based on a 5-point Likert scale from 1 (not useful at all; unlikely) to 5

Figura 6: Gráfico sobre a utilidade da interface Fonte: Chen e Choi, 2006

Na conclusão os pesquisadores observam que os participantes entendem que o processo lógico de localização das fontes foi identificado, porém alguns argumentaram que costumam selecionar as fontes também considerando sua relação com sentimentos e humores.

Os resultados do projeto indicam que quando os participantes têm todo o conjunto de caracteres disponíveis, a maioria deles pode usar a Identifont para encontrar fontes com sucesso. No entanto, esta situação não é um cenário ideal pois muitas vezes os designers gráficos não têm um conjunto completo de caracteres. Preencher esta lacuna entre os caracteres em falta e a abordagem de perguntaresposta de ferramentas de localização de fonte é o próximo passo para a melhoria do sistema da interface.

Outro ponto desfavorável apresentado pelos participantes é que ao final da tarefa muitas vezes a interface ainda apresentava diversas opções de fonte, quando o esperado pelos usuários era a indicação de apenas uma fonte correta. (very useful; very likely); $\mathrm{N}=35$
Realização:
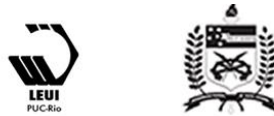


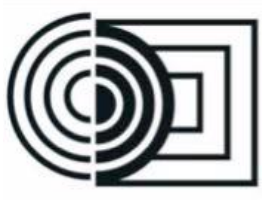

$\mathrm{O}$ terceiro artigo analisado trata sobre o

desenvolvimento de um menu de seleção intuitivo centrado no usuário. A técnica utilizada na pesquisa foi o cardsorting e o objetivo era analisar como os indivíduos classificam fontes Devanagari, a escrita usada na Índia e no Nepal.

O autor destaca a importância do menu de seleção como elemento de interface nos softwares ressaltando que pesquisadores como Lee e Raymond (1993) já consolidaram resultados sobre o tema, porém não foram identificados estudos específicos sobre menus de seleção de fontes tipográficas. (DALVI, 2010)

Os estudos sobre menus sugerem que é preciso um equilíbrio entre amplitude e profundidade, ou seja, o menu não deve ter tantas opções, muito amplo, para que a busca não seja demorada nem deve ser tão segmentado, com muitas hierarquias, para que o usuário não se perca em meio a tantas decisões. Importante observar que embora o artigo analisado seja de 2010 as referências sobre menu citadas são das décadas de 1980 e 1990.

Rosh (1973 apud DALVI, 2010) sugere que as categorias de menu devem refletir a estrutura cognitiva dos usuários não-especialistas, ou seja, de quem realmente irá usar a ferramenta e não dos seus desenvolvedores. Dalvi (2010) destaca ainda em seu artigo que a confiabilidade das categorias criadas aumenta a medida em que mais usuários são consultados e a categorização técnica é aprimorada.

A pesquisa foi realizada com 38 participantes, masculinos e femininos com idades entre 21 e 42 anos, com um nível mínimo de formação e alfabetizados no idioma em questão. Eles receberam cartões de $25 \mathrm{x} 4 \mathrm{~cm}$ com textos Devanagari em corpo 72 pontos, como mostra a figura 7. $16^{\circ}$ Ergodesign - Congresso Internacional de Ergonomia e Usabilidade de Interfaces Humano Tecnológica: Produto, Informações Ambientes Construídos e Transporte

$16^{\circ}$ USIHC - Congresso Internacional de Ergonomia e Usabilidade de Interfaces Humano Computador

CINAHPA | 2017 - Congresso Internacional de Ambientes Hipermídia para Aprendizagem.

\section{झिखृफुद्धी बुठूभीश् किट्रेन्न आंग्ररे}

\section{झिखृफुद्धी बुठूभीश् किट्रेन्न आंग्रचै}

\section{झिखृफुद्धी बुटूमीश् किट्रेर्न आंग्रै}

\section{झिखृफुद्धी बुठूभीश् किट्रेर्न आंग्रै}

Figura 7: Cartões com fontes Devanagari usados na pesquisa.

Fonte: Dalvi (2010)

A primeira etapa do exercício consistiu em uma fase de aquecimento, quando os participantes receberam 33 cartões com nomes de animais e foram convidados a organizá-los em categorias da forma que achassem mais adequado. Com isso os pesquisadores buscaram se assegurar de que os participantes não tinham dúvidas sobre o processo de classificação.

$\mathrm{Na}$ etapa seguinte os participantes receberam 30 cartões com amostras de fontes e da mesma forma foram convidados a organizá-las em categorias e subcategorias de acordo com a sua percepção, podendo colocar a mesma fonte em mais de uma categoria. Depois foram informados de que deveriam dar nomes as classes que haviam criado, explicá-las e definir suas propriedades. Concluída esta etapa os grupos puderam rever suas classificações, corrigir o que considerassem necessário e então registrar em uma folha de coleta de dados as categorias e suas propriedades.

Nos resultados o artigo apresenta os principais agrupamentos criados pelos participantes, onde percebe-se que a questão formal é predominante. As fontes foram agrupadas pelo tipo do traço, pelas aberturas, presença de serifas, aplicação - fontes para destaque/display. As fontes também foram sub-agrupadas de acordo com o peso e a largura.

A partir da análise dos dados foram definidas 5 categorias principais: Texto Tradicional, Script, Serifa, Monolinear e Display. O autor finaliza observando que o estudo produziu uma grande quantidade de dados além do que foi apresentado 


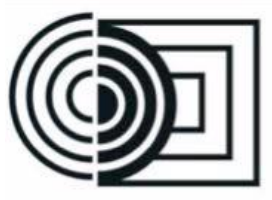

no artigo e que testes de usabilidade das categorias ainda seriam necessários (DALVI, 2010).

A pesquisa apresentada no artigo de fez parte da tese do autor e resultou em uma ferramenta web para pesquisa de fontes Devanagari bastante intuitiva. Os atributos das fontes podem ser selecionados visualmente e foram organizados em 12 itens que se referem basicamente as formas, traços e proporções da fonte, como mostram as figuras 8 e 9 . O site onde se encontra a interface traz ainda uma descrição detalhada da terminologia usada, facilitando assim a compreensão por usuários iniciantes. Muito oportuno uma vez que a interface se destina também a estudantes.

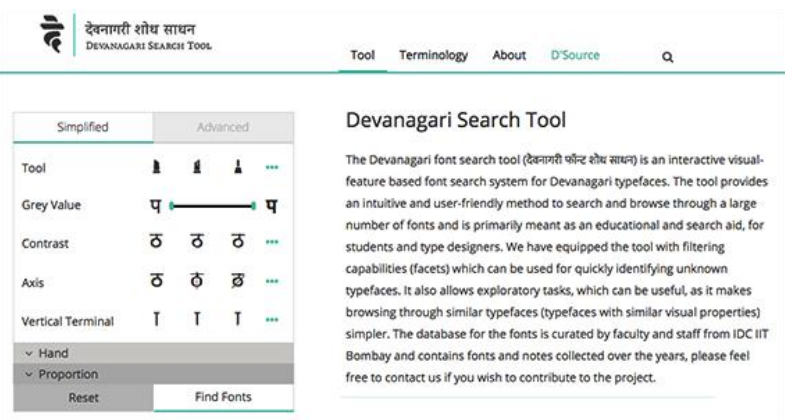

Figura 8: Tela inicial do site sobre a interface de pesquisa de fontes Devanagari Disponível em: http://dsquare.in/devft/en/index.php.

\begin{tabular}{|c|c|c|c|c|}
\hline Simplified & \multicolumn{4}{|c|}{ Advanced } \\
\hline Tool & $\mathbf{E}$ & 重 & ! & ... \\
\hline Grey Value & प & & & 4 \\
\hline Contrast & ठ & ठ & ठ & ... \\
\hline Axis & ठ & $\bar{\phi}$ & $\bar{\varnothing}$ & $\ldots$ \\
\hline Vertical Terminal & $\mathrm{T}$ & $\mathrm{T}$ & $\mathrm{T}$ & $\bullet$ \\
\hline \multicolumn{5}{|l|}{$\checkmark$ Hand } \\
\hline \multicolumn{5}{|l|}{$\checkmark$ Proportion } \\
\hline Reset & & Fin & Fonts & \\
\hline
\end{tabular}

$16^{\circ}$ Ergodesign - Congresso Internacional de Ergonomia e Usabilidade de Interfaces Humano Tecnológica: Produto, Informações Ambientes Construídos e Transporte

$16^{\circ}$ USIHC - Congresso Internacional de Ergonomia e Usabilidade de Interfaces Humano Computador

CINAHPA | 2017 - Congresso Internacional de Ambientes Hipermídia para Aprendizagem.

Figura 9: Detalhe do menu de seleção baseado nos atributos formais dos caracteres.

Disponível em: http://dsquare.in/devft/en/index.php.

\subsection{Discussões}

Comparando os 3 artigos analisados percebe-se que as duas últimas interfaces digitais enfatizam a questão formal da letra, ou seja, oferecem ao usuário a possibilidade de escolher a fonte de acordo com atributos como peso, contraste, presença ou não de serifas, formato de alguns caracteres, entre outro elementos. Já a primeira interface categoriza as fontes de acordo com sua personalidade: calma, delicada, forte, desleixada, feliz, aliando também a classificação tradicional: display, sem serifa, moderna, entre outras.

Outra característica interessante da primeira interface para seleção de fontes, é que ela mostra opções de layout para que o usuário escolha a fonte a partir de um contexto, o que é pertinente. De acordo com Bringhurst (2005) a escolha das fontes deve ser relacionada à tarefa $\mathrm{e}$ ao assunto. $\mathrm{O}$ autor considera relevante que uma fonte apresente ornamentos e fantasias que remetam à temática do projeto, porém destaca que a qualidade e a legibilidade, nesta ordem, são mais importantes.

Considerando os 8 critérios apresentados no quadro 1 - legibilidade, recursos e variações, aspectos histórico-culturais, expressão, qualidade, suporte, licenciamento e investimento resultantes da revisão bibliográfica, observa-se que estes não aparecem explicitamente nas interfaces digitais analisadas. No entanto, os recursos que favorecem a busca por atributos e personalidade, presentes nas 3 interfaces, são úteis para filtrar fontes que atendessem aos critérios da legibilidade e da expressão, por exemplo.

O critério "recursos e variações", considerado muito importante principalmente em projetos editoriais, não é contemplado pela busca de nenhuma das interfaces. Um filtro por família poderia auxiliar o designer na tarefa de encontrar grupos de fontes com características semelhantes e variações necessárias para atender as demandas. 


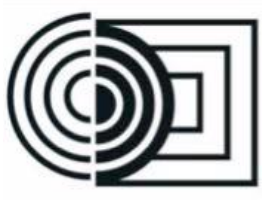

De uma forma geral considera-se que as interfaces analisadas apresentam recursos interessantes de busca e seleção porém nenhuma delas auxilia efetivamente o usuário na tomada de decisão, ou seja, em definir quais são as características da fonte mais adequadas ao seu projeto. É importante destacar que esta tomada de decisão pode ser complexa e ainda não é possível afirmar se uma interface digital poderia efetivamente auxiliar neste processo. De acordo com Lupton (2013 p. 28)

Ao escolher as fontes, os designers gráficos consideram a história dos tipos e suas conotações atuais, bem como suas qualidades formais. $\mathrm{O}$ objetivo é encontrar uma combinação apropriada entre o estilo das letras, a situação social específica e a massa de conteúdo que definem o projeto. Nenhuma cartilha é capaz de fixar o significado ou a função de cada fonte, cada designer deve enfronhar-se nessa biblioteca de possibilidades à luz das circunstâncias únicas de cada projeto. (LUPTON, 2013 p. 28)

Ainda segundo Fontoura e Fukushima (2012), ao escolher uma fonte é importante definir o que vai ser lido, ou seja, a quantidade de texto, qual a sua função, se é informativo, instrutivo ou para entretenimento, quem vai ler e em quais circunstâncias e ambiente. Nenhuma das interfaces analisadas tem foco neste processo de decisão, elas apenas apoiam o usuário a encontrar uma determinada fonte, ou estilo de fonte, previamente definida.

\section{Considerações finais}

Com a conclusão da pesquisa constatou-se que a revisão integrativa foi apropriada para identificar estudos sobre interfaces digitais de seleção tipográfica. Devido a sua sistematização poderá ser repetida futuramente a fim de identificar novos estudos publicados e manter a pesquisa atualizada.

Embora apenas 3 artigos tenham sido considerados relevantes, estes trouxeram significativas contribuições para a pesquisa. As interfaces analisadas ou propostas pelos autores demonstram que é possível realizar a seleção tipográfica de forma mais detalhada, considerando atributos formais e de personalidade da fonte. Porém, comparando os recursos de seleção oferecidos $16^{\circ}$ Ergodesign - Congresso Internacional de Ergonomia e Usabilidade de Interfaces Humano Tecnológica: Produto, Informações Ambientes Construídos e Transporte

$16^{\circ}$ USIHC - Congresso Internacional de Ergonomia e Usabilidade de Interfaces Humano Computador

CINAHPA | 2017 - Congresso Internacional de Ambientes Hipermídia para Aprendizagem.

pelas interfaces com os critérios de seleção preliminares definidos na revisão bibliográfica constatou-se que as interfaces não contemplam todos os critérios. Não é possível, por exemplo, selecionar por meio destas interfaces fontes de acordo com suas regras de licenciamento, extensão da família e presença de caracteres específicos.

Cabe destacar ainda que das 3 interfaces estudadas nos artigos apenas 1 está funcionando efetivamente e pode ser usada em contextos de projeto, a Identifont. Entende-se, portanto, que existe demanda para mais pesquisas que apontem para uma solução digital efetiva no auxílio ao processo de seleção de fontes atendendo a critérios mais abrangentes.

\section{BIBLIOGRAFIA}

ALI, Fatima. A arte de editar revistas. São Paulo: Companhia Editora Nacional, 2009.

BEIER, Sofie. Typeface Legibility: Towards defi ning familiarity. 2009. 268f. Tese (Doutorado) Curso de Filosofia, The Royal College Of Art, Londres, 2009.

BOTELHO, Louise Lira Roedel; CUNHA, Cristiano Castro de Almeida; MACEDO, Marcelo. O Método da Revisão Integrativa nos Estudos Organizacionais. Gestão e Sociedade, Belo Horizonte, v. 5, n. 11, p. 121-136, 2011.

BRINGHURST, Robert. Elementos do Estilo Tipográfico. São Paulo: Cosac Naify, 2005.

CHEN , H.-L; CHOI, G. An online font library: evaluation by graphic design students. The Electronic Library 24, 774-790, 2006.

DALVI, Girishi. Development of an intuitive usercentric font selection menu. In IFIP Advances in Information and Communication Technology, pp. 144-153, 2010.

FARIAS, Priscila Lena. Tipografia Digital: o impacto das novas tecnologias. $4^{\mathrm{a}}$ ed. Teresópolis: 2AB, 2013. 


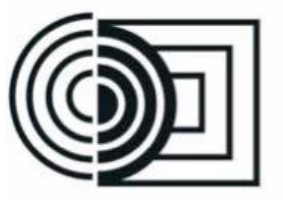

FONTOURA, Antônio M. e FUKUSHIMA, Naotake. Vade-mécum de Tipografia. Editora Insight, 2012.

FRUTIGER, Adrian. Sinais \& Símbolos : Desenho, projeto e significado. 2.ed. São Paulo: Martins Fontes, 2007.

HASLAM, Andrew. O livro e o designer II Como criar e produzir livros. São Paulo: Edições Rosari, 2007.

HENDEL, Richard. O design do livro. São Paulo: Ateliê Editorial, 2003.

HENESTROSA, Cristobal; MESEGUER, Laura; SCAGLIONE, José. Como criar tipos: do esboço à tela. Brasília: Estereográfica, 2014.

JURY, David. O que é a Tipografia. Portugal: Gustavo Gili, 2006.

LEEUWEN, Theo van. Towards a semiotics of Typography. Information Design Journal + Document Design 14(2), 139-155. 2006 John Benjamins Publishing Company

LUPTON, Ellen. Pensar com tipos: um guia para designers, escritores, editores e estudantes. São Paulo: Cosac Naify, 2013.

O'DONOVAN, Peter; LÏBEKS, Jānis; AGARWALA, Aseem; HERTZMANN, Aaron. Exploratory font selection using crowdsourced attributes. Jornal of ACM Transactions on Graphics, Vol. 33, No. 4, Article 92, 2014.

O'DONOVAN, Peter. Learning Design: Aesthetic Models for Color, Layout and Typography. Tese (doutorado). Departamento de Ciência da Computação. Universidade de Toronto, 2015.

PAMENTAL, Jason. Responsive Typography: Using Type Well on the Web. Sebastopol: O’Reilly Media, 2014.

ROCHA, Claudio. Novo Projeto Tipográfico: análise e produção de fontes digitais. São Paulo: Rosari, 2012. $16^{\circ}$ Ergodesign - Congresso Internacional de Ergonomia e Usabilidade de Interfaces Humano Tecnológica: Produto, Informações Ambientes Construídos e Transporte

$16^{\circ}$ USIHC - Congresso Internacional de Ergonomia e Usabilidade de Interfaces Humano Computador

CINAHPA | 2017 - Congresso Internacional de Ambientes Hipermídia para Aprendizagem.

SAMARA, Timothy. Evolução do design: da teoria à prática. Porto Alegre: Bookman, 2010.

SHAIK, Audrey Dawn. Psychology of onscreen type: investigations regarding typeface personality, appropriateness, and impact on document perception. 2007. Tese (doutorado) 2007. Departamento de Psicologia, Wichita State University, College of Liberal Arts and Sciences, Kansas, 2007.

SMEIJERS, Fred. Contrapunção: fabricando tipos no século dezesseis, projetando tipos hoje.

Brasília: Estereográfica: 2015.

UNGER, Gerard. Enquanto você lê. Brasília: Estereográfica, 2016.

ZAPATERRA, Yolanda. Design Editorial. São Paulo: Gustavo Gili, 2014.

UNGER, Gerard. Enquanto você lê. Brasília:

Estereográfica, 2016.

WHITTEMORE, R.; KNAFL, K. The integrative review: updated methodology. Journal of Advanced Nursing, Oxford, v. 52, n. 5, p. 546-553, Dec. 2005. 